PROCEEDINGS OF THE

AMERICAN MATHEMATICAL SOCIETY

Volume 131, Number 12, Pages 3777-3781

S 0002-9939(03)06965-X

Article electronically published on February 24, 2003

\title{
ON COMMUTING OPERATOR EXPONENTIALS
}

\author{
FOTIOS C. PALIOGIANNIS
}

(Communicated by Joseph A. Ball)

\begin{abstract}
Let $A, B$ be bounded operators on a Banach space with $2 \pi i$ congruence-free spectra such that $e^{A} e^{B}=e^{B} e^{A}$. E. M. E. Wermuth has shown that $A B=B A$. Ch. Schmoeger later established this result, using inner derivations and, in a second paper, has shown that: for $a, b$ in a complex unital Banach algebra, if the spectrum of $a+b$ is $2 \pi i$-congruence-free and $e^{a} e^{b}=e^{a+b}=e^{b} e^{a}$, then $a b=b a$ (and thus, answering an open problem raised by E. M. E. Wermuth). In this paper we use the holomorphic functional calculus to give alternative simple proofs of both of these results. Moreover, we use the Borel functional calculus to give new proofs of recent results of Ch. Schmoeger concerning normal operator exponentials on a complex Hilbert space, under a weaker hypothesis on the spectra.
\end{abstract}

Let $X$ be a Banach space and $\mathcal{A}=B(X)$ the Banach algebra of all bounded operators on $X$. For $A \in \mathcal{A}$ the spectrum of $A$ is denoted by $\sigma(A)$. We assume that the reader is familiar with the holomorphic functional calculus (see, e.g., 1, Chap. VII] or [2 Chap. 3]). We denote by $\mathcal{H}(A)$ the algebra of functions holomorphic in a neighborhood of $\sigma(A)$.

The $\sigma(A)$ is said to be $2 \pi i$-congruence free if $\sigma(A) \cap \sigma(A+2 k \pi i)=\varnothing$ for $k= \pm 1, \pm 2, \ldots$

The following result is due to E. M. E. Wermuth (8] ). We give here an alternative proof. See also Ch. Schmoeger ([5]) for a short proof using inner derivations.

Theorem 1. Let $A, B \in \mathcal{A}$. Suppose that $\sigma(A)$ and $\sigma(B)$ are $2 \pi i$-congruence-free and that $e^{A} e^{B}=e^{B} e^{A}$. Then $A B=B A$.

Proof. Setting $T=e^{B}$, the hypothesis becomes $e^{A} T=T e^{A}$. We show that $A T=$ $T A$. Since $\sigma(A)$ is compact and $2 \pi i$-congruence-free, there is $\delta>0$ such that with $D=\{z \in \mathbf{C}:|z-\lambda|<\delta$ for some $\lambda \in \sigma(A)\}$ we have $D \cap \bigcup_{k= \pm 1, \pm 2, \ldots}(D+2 k \pi i)=\varnothing$ and $\sigma(A) \subseteq D$.

Now for $z \in D$, the exponential function $f(z)=e^{z}$ is one-to-one holomorphic in $D$, and therefore has a holomorphic inverse $g: e^{D} \rightarrow D$ (e.g., 4, Thm. 1033]) such that $g(f(z))=z$. At the same time, by the spectral mapping theorem we have $\sigma\left(e^{A}\right)=\left\{e^{\lambda}: \lambda \in \sigma(A)\right\}$. Since, $e^{A} T=T e^{A}$ and $g \in \mathcal{H}\left(e^{A}\right)$ it follows (e.g., [1, Chap. VII, Prop. 4.9]) that $g\left(e^{A}\right) T=T g\left(e^{A}\right)$. Moreover, since $g \circ f=i d$ on $\sigma(A)$ (where $i d(\lambda)=\lambda \forall \lambda \in \sigma(A))$ and $g(f(A))=(g \circ f)(A)$ (see, e.g., [2, Thm. 3.3.8]), we have $g\left(e^{A}\right)=A$. Thus, $A T=T A$.

Received by the editors April 8, 2002 and, in revised form, July 2, 2002.

2000 Mathematics Subject Classification. Primary 47A60.

Key words and phrases. Commuting exponentials, holomorphic functional calculus, Borel functional calculus. 
To finish the proof of the theorem, note now that, since $A e^{B}=e^{B} A$ and $\sigma(B)$ is $2 \pi i$-congruence-free, the holomorphic functional calculus applied to $B$ and a similar argument as above give $A B=B A$.

We now give a simpler proof of a result due to Ch. Schmoeger ([ 6$]$ ) that answers the following open problem raised by E. M. E. Wermuth in [8]: To find a natural assumption which together with $e^{A} e^{B}=e^{A+B}$ implies $A B=B A$.

Theorem 2. Let $A, B \in \mathcal{A}$. Suppose that $\sigma(A+B)$ is $2 \pi i$-congruence-free. If $e^{A} e^{B}=e^{A+B}=e^{B} e^{A}$, then $A B=B A$.

Proof. Set $S=A+B$ and $T=e^{A}$. Then we have $T e^{B}=e^{S}=e^{B} T$. This implies $e^{S} T=T e^{S}\left(=T e^{B} T\right)$. Since the spectrum of $S$ is $2 \pi i$-congruence-free, a similar argument as in the proof of Theorem 1 gives that $S T=T S$. Moreover, since the map $f \mapsto f(A)$ of $\mathcal{H}(A) \rightarrow \mathcal{A}$ is an algebra homomorphism, we have $A e^{A}=e^{A} A$.

Now, $(A+B) e^{A}=e^{A}(A+B)$ implies that $B e^{A}=e^{A} B$. Multiplying this last equality to the left by $e^{B}$ (and noting that $e^{B} B=B e^{B}$ ) we get $e^{B} B e^{A}=$ $B e^{B} e^{A}=e^{B} e^{A} B$, which, by using the hypothesis, gives $B e^{S}=e^{S} B$. Again the proof of Theorem 1 implies that $S B=B S$. Therefore, $A B=B A$ and the proof is complete.

Next, we give an alternative proof of two further results of Schmoeger ([6 Thm. $5(\mathrm{a})$ and Corollary 2]).

Theorem 3. Let $A, B \in \mathcal{A}$. Suppose that $\sigma(A)$ is $2 \pi i$-congruence-free and $e^{A}=e^{B}$. Then $A \in\{B\}^{\prime \prime}$ (and so $A B=B A$ ).

Proof. Let $T$ be any operator that commutes with $B$, i.e., $T B=B T$. Then $T f(B)=f(B) T$ for any $f \in \mathcal{H}(B)$. In particular, $T e^{B}=e^{B} T$. As $e^{A}=e^{B}$, we have $T e^{A}=e^{A} T$.

Now, since $\sigma(A)$ is $2 \pi i$-congruence-free, it follows, as before, that $T A=A T$. Therefore, $A \in\{B\}^{\prime \prime}$ (the double commutant of $B$ ), and so $A B=B A$.

Corollary 4. Let $A, B$ be bounded self-adjoint operators on a complex Hilbert space. If $e^{A}=e^{B}$, then $A=B$.

Proof. Since $\sigma(A) \subseteq \mathbf{R}, \sigma(A)$ is automatically $2 \pi i$-congruence-free (and so is $\sigma(B)$ ). By Theorem 3 we have $A B=B A$. It now follows that $I=e^{A}\left(e^{B}\right)^{-1}=e^{A} e^{-B}=$ $e^{A-B}$. Let $S=A-B$ and $\mathcal{U}=\{S\}^{\prime \prime}$ be the abelian $C^{*}$-algebra generated by self-adjoint operator $S$. If - denotes the Gelfand isomorphism from $\mathcal{U}$ onto $C(Y)$, where $Y$ is the maximal ideal space of $\mathcal{U}$, then (from the continuous functional calculus) we have

$$
e^{S}=I \Rightarrow\left(\widehat{e^{S}}\right)=1 \Rightarrow e^{\widehat{S}}=1=e^{0} \quad \text { on } \sigma(S) \subseteq \mathbf{R} .
$$

Therefore, $\widehat{S}=0$, and so $A=B$.

Now, we turn our attention to normal operator exponentials. Let $B(\mathcal{H})$ be the algebra of all bounded operators on a complex Hilbert space $\mathcal{H}$. Let $N \in B(\mathcal{H})$ be a normal operator and let $N=\int \lambda d E(\lambda)$ be its spectral decomposition, where $E$ is the associated spectral measure defined on the Borel subsets of the spectrum of $N, \sigma(N)$. We denote by $B(\sigma(N))$ the $C^{*}$-algebra of all bounded Borel measurable complex-valued functions on $\sigma(N)$, and by $\Phi$ the *-homomorphism $\Phi(f)=f(N)=$ $\int f d E$, from $B(\sigma(N))$ to $\{N\}^{\prime \prime}$ - the abelian von Neumann algebra generated by 
$N$. This is the Borel functional calculus for $N$ (see, e.g., [1, Chap. IX, Thm. 2.3] or [3 Thm. 3.3, Thm. 3.10]).

Commuting normal operator exponentials are studied in a recent paper of $\mathrm{Ch}$. Schmoeger ([7]). In [7], the spectrum $\sigma(N)$ is defined to be generalized $2 \pi i$ congruence-free if

$$
E(\sigma(N) \cap \sigma(N+2 k \pi i))=0 \text { for all } k=1,2, \ldots
$$

Note that since $E(\varnothing)=0$, if $\sigma(N)$ is $2 \pi i$-congruence-free, then $\sigma(N)$ is generalized $2 \pi i$-congruence-free.

Under this hypothesis of generalized $2 \pi i$-congruence-free spectra, Ch. Schmoeger proves a series of results concerning normal operator exponentials (see [7, Theorems $1.2,1.3,1.4,1.5])$.

In the sequel, we give new direct proofs of Schmoeger's results under a weaker hypothesis on the spectra. In fact, as it was suggested to the author by the anonymous referee, Schmoeger's definition of generalized $2 \pi i$-congruence-free spectrum may be revised in a weaker form that reads as follows:

Definition. Let $N \in B(\mathcal{H})$ be a normal operator and let $E$ be its spectral measure. The spectrum $\sigma(N)$ is said to be generalized $2 \pi i$-congruence-free if there is a Borel subset $\Delta$ of $\sigma(N)$ such that $E(\Delta \cap(\Delta+2 k \pi i))=0$ for all $k=1,2, \ldots$ and $E(\sigma(N) \backslash \Delta)=0$.

Remark 5. Note that this revised version of Schmoeger's definition is a weaker hypothesis on the $\sigma(N)$; for if $E(\sigma(N) \cap \sigma(N+2 k \pi i))=0$ for all $k=1,2, \ldots$, then, since $\sigma(N)$ is compact, the set $\Delta=\sigma(N) \backslash \bigcup_{k=1}^{m}(\sigma(N) \cap \sigma(N+2 k \pi i))$ is a Borel (relatively open) subset of $\sigma(N)$ such that $E(\Delta \cap(\Delta+2 k \pi i))=0$ for all $k=1,2, \ldots$ and $E(\sigma(N) \backslash \Delta)=0$.

Through the rest of this paper the hypothesis of generalized $2 \pi i$-congruence-free spectrum is to be understood in the sense of the above definition.

Theorem 6. Let $N, M$ be bounded normal operators in $B(\mathcal{H})$ and suppose that $e^{N} e^{M}=e^{M} e^{N}$.

(a) If $\sigma(N)$ is generalized $2 \pi i$-congruence-free, then $N e^{M}=e^{M} N$.

(b) If $\sigma(N)$ and $\sigma(M)$ are generalized $2 \pi i$-congruence-free, then $N M=M N$.

Proof. (a) Set $T=e^{M}$. Then $e^{N} T=T e^{N}$. We prove that $N T=T N$. Since $\sigma(N)$ is generalized $2 \pi i$-congruence-free, there is a Borel subset $\Delta$ of $\sigma(N)$ such that $E(\Delta \cap(\Delta+2 k \pi i))=0$ for all $k=1,2, \ldots$ and $E(\sigma(N) \backslash \Delta)=0$.

Let $\Omega=\Delta \backslash \bigcup_{k \neq 0}(\Delta \cap(\Delta+2 k \pi i))$. Then the exponential function $f(z)=e^{z}$ is one-to-one on $\Omega$ and $E(\Omega)=I$. If $\alpha=\sup _{z \in \sigma(N)}\left|e^{z}\right|$ and $\beta=\sup _{z \in \sigma(N)}|z|=r(N)$ (the spectral radius of $N$ ), choose $z_{0} \in \mathbf{C}$ such that $\left|z_{0}\right|>\alpha+\beta$ and define a function $\tilde{f}$ on $\sigma(N)$ by

$$
\tilde{f}(z)= \begin{cases}f(z) & \text { if } z \in \Omega, \\ z+z_{0} & \text { if } z \in(\sigma(N) \backslash \Omega) .\end{cases}
$$

The function $\tilde{f}$, so defined, is a bounded Borel function which is one-to-one on $\sigma(N)$, and therefore has an inverse function $g$ from $\tilde{f}(\sigma(N))$ to $\sigma(N)$ such that $g(\tilde{f}(z))=z$. It is easy to see that the direct image under $\tilde{f}$ of any Borel set in $\sigma(N)$ is a Borel set, and so $g$ is a (bounded) Borel function on $\tilde{f}(\sigma(N))$. At the 
same time, since $E(\sigma(N) \backslash \Omega)=0$ and $\tilde{f}-f=\chi_{\sigma(N) \backslash \Omega} \cdot(\tilde{f}-f)$, it follows that $\Phi(\tilde{f}-f)=0$. Hence, $\tilde{f}(N)=f(N)=e^{N}$.

Moreover, since $\sigma\left(e^{N}\right)=\sigma(\tilde{f}(N)) \subseteq \overline{\tilde{f}(\sigma(N))}$ ([3 Prop. 3.6]), no matter how one extends $g$ on $\bar{f}(\sigma(N))$, we have that $g \in B\left(\sigma\left(e^{N}\right)\right)$ and $g(\tilde{f}(\lambda))=\lambda \forall \lambda \in \sigma(N)$. It follows now by, e.g., [3, Prop. 3.7] that $g\left(e^{N}\right)=N$.

Furthermore, since $e^{N}$ is normal and $e^{N} T=T e^{N}$, the spectral theorem ([1) Chap. IX, Prop. 8.1] or [3, Thm. 3.10]) implies $g\left(e^{N}\right) T=T g\left(e^{N}\right)$. Thus, $N T=$ $T N$.

(b) From (a) we have $N e^{M}=e^{M} N$. Since $M$ is normal and $\sigma(M)$ is generalized $2 \pi i$-congruence-free, an analogous argument as above gives $N M=M N$.

The following example, which was provided by the anonymous referee, shows that Schmoeger's generalized $2 \pi i$-congruence-free hypothesis indeed needs to be relaxed.

Example 7. Let $\mu=\lambda+\nu$ where $\lambda$ is Lebesgue measure on the unit interval $[0,1]$ and $\nu$ is a singular measure on the interval $[2 \pi i, 1+2 \pi i]$, and let $N=M_{z}$ (multiplication by the coordinate function $z$ ) on $L^{2}(\mu)$. Then

$$
\mu(\sigma(N) \cap \sigma(N+2 \pi i))=\mu([2 \pi i, 1+2 \pi i])>0 .
$$

At the same time there exists a set $\Omega \subset \sigma(N)$ such that the exponential function is one-to-one on $\Omega$ and $\mu(\mathbf{C} \backslash \Omega)=0$. Thus, in this case, Theorem [6) ([7] Thm. $1.2(\mathrm{a})])$ is valid even though Schmoeger's hypothesis is violated. $1.5])$.

We conclude this paper by proving the remaining results ( 7 , Theorems 1.3, 1.4,

Theorem 8. Let $A, B \in B(\mathcal{H})$. Suppose that $A+B$ is normal and $\sigma(A+B)$ is generalized $2 \pi i$-congruence-free. If $e^{A} e^{B}=e^{A+B}=e^{B} e^{A}$, then $A B=B A$.

Proof. Set $N=A+B$ and $T=e^{A}$. Reasoning as in Theorem 2 and as in the proof of Theorem 6(a), the result follows.

Theorem 9. Let $A \in B(\mathcal{H})$ be normal and suppose that $\sigma(A)$ is generalized $2 \pi i$ congruence-free. If $B \in B(\mathcal{H})$ and $e^{A}=e^{B}$, then $A \in\{B\}^{\prime \prime}($ and so $A B=B A$ ).

Proof. Reason as in Theorem 3 and use the normality of $A$ and the generalized $2 \pi i$-congruence-freedom of its spectrum.

Theorem 10. Let $A, B \in B(\mathcal{H})$. If $A$ is self-adjoint, $\sigma(A) \subset[-\pi, \pi]$ and $e^{i A}=e^{B}$, then we have

(a) $B^{*}=-B$ if $B$ is normal,

(b) $A \in\{B\}^{\prime \prime}$, if $\pi$ or $-\pi$ is not an eigenvalue of $A$.

Proof. Argue as in [7, Theorem 1.5]; for part (a) use Corollary 4, and for part (b) use Remark 5 and Theorem 9 .

\section{ACKNOWLEDGMENT}

The author would like to thank the anonymous referee for suggesting the weaker version of the generalized $2 \pi i$-congruence-free hypothesis and for providing Example 7] 


\section{REFERENCES}

[1] J. B. Conway, A Course in Functional Analysis, Springer-Verlag, New York, 1985. MR 86h:46001

[2] R. V. Kadison and J. R. Ringrose, Fundamentals of the theory of operator algebras, Acad. Press, New York, 1983. MR 85j:46099

[3] F. C. Paliogiannis, Topological function-theoretic proofs in spectral theory, Rend. Circ. Mat. Palermo, Seri II Tomo XLIV (1995), 21-44. MR 96e:47027

[4] W. Rudin, Real and Complex Analysis, McGraw-Hill, New York, 1974. MR 49:8783

[5] Ch. Schmoeger, Remarks on commuting exponentials in Banach algebras. Proc. Amer. Math. Soc. 127, no. 5 (1999), 1337-1338. MR 99h:46090

[6] Ch. Schmoeger, Remarks on commuting exponentials in Banach algebras, II. Proc. Amer. Math. Soc. 128, no. 11 (2000), 3405-3409. MR 2001b:46077

[7] Ch. Schmoeger, On normal operator exponentials, Proc. Amer. Math. Soc. 130, no. 3 (2002), 697-702. MR 2002j:47032

[8] E. M. E. Wermuth, A remark on commuting operator exponentials, Proc. Amer. Math. Soc. 125, no. 6 (1997), 1685-1688. MR 97g:39011

Department of Mathematics, St. Francis College, 180 Remsen Street, Brooklyn, NEW York 11201

E-mail address: fpaliogiannis@stfranciscollege.edu 